\title{
Transitions to Chaos and Chaos Synchronization for Solitons in the Presence of Spatially Inhomogeneous Forces
}

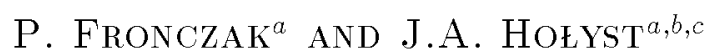 \\ ${ }^{a}$ Faculty of Physics, Warsaw University of Technology \\ Koszykowa 75, 00-662 Warsaw, Poland \\ ${ }^{b}$ Max-Planck Institute for Physics of Complex Systems \\ Nöthnitzer Straße 36, 01187 Dresden, Germany \\ ${ }^{c}$ Institute for Economics and Traffic, Dresden University of Technology \\ Andreas-Schubert-Straße 23, 01062 Dresden, Germany
}

(Received December 31, 2001)

\begin{abstract}
Motion of kink solitons in the $\varphi^{4}$ model in the presence of external spatially inhomogeneous forces is studied. Depending on the system parameters various routes to chaos, i.e. Feigenbaum's scenario, type-I intermittency, and chaos-chaos intermittency are observed. Synchronization of chaotic solitons is investigated.
\end{abstract}

PACS numbers: 05.45.Pq, 05.45.Yv

\section{Introduction}

Recently there has been a growing interest in soliton dynamics in inhomogeneous media. Early papers considered structureless solitons and treated them as point-like particles interacting with delta-function-like impurities [1,2] but real systems involve finite-width inhomogeneity when the spatially extended character of the soliton has to be taken into account [3-6].

Rich dynamics of such "extended" soliton systems influenced by additional perturbations occurs in various models. Examples are Josephson junctions [7, 8], optical systems $[9,10]$ or plasma models [11]. Furthermore, spatial properties of solitons and impurities were considered in several papers [4, 12-14] from numerical and analytical points of view and many unexpected phenomena were observed (e.g. the soliton explosions [4]). One of the common features of the perturbed 
soliton systems is the transition to chaos [12-16]. The phenomenon usually occurs in the presence of an external field which pumps energy into the system, influences internal soliton modes and can finally lead to soliton destruction. The chaotic behaviour was also observed in cooperative motions of solitons in the lattice formed by five coupled chains [16]. In [17] we used the time-delay feedback method [18-20] to suppress the chaotic soliton motion.

In the present paper we investigate transitions to chaos of soliton motion in a one-dimensional driven damped $\varphi^{4}$ model [21] in the presence of a phase boundary. We show that two such systems can be synchronized by a proper coupling.

Let us consider a model of a one-dimensional classical scalar field $\varphi(x, t)$ governed by the equation of motion

$$
\varphi_{x x}-\varphi_{t t}-\gamma \varphi_{t}+\frac{1}{2} \varphi-\frac{1}{2} \varphi^{3}=-F(x)-G(x, t),
$$

where $\gamma>0$ is a damping constant, the static force $F(x)$

$$
F(x)=B\left(4 B^{2}-1\right) \tanh (B x)
$$

represents a typical phase boundary centred at the point $x=0$ and $G(x, t)$ is a pumping force. For $G(x, t)=0$ there is a static soliton solution of (1) in the form of the kink

$$
\varphi_{k}(x, t)=2 B \tanh (B x)
$$

which is pinned by the force $F(x)$ to the site $x=0$. The linear stability of this solution was analyzed in [3] and it was proved that in the limit of zero damping the kink is stable provided that $B>1 / 2$. We choose the space-time dependent pumping force $G(x, t)$ in such a way that its spatial part corresponds exactly to the ground state function of the operator describing small oscillations (linear phonons) around the kink $[22,23]$, i.e.

$$
G(x, t)=P \cos \left(\omega_{0} t\right) \cosh ^{-2}(B x) .
$$

Since the force $G(x, t)$ is proportional to the first derivative of the kink shape $\varphi_{k}(x)$ thus in the limit of small amplitudes $P \ll 1$ it pumps energy mostly into the translational mode of the kink (3) and it shifts the position of the kink centre without large disturbances of the kink shape. This effect is limited however only to the case when the kink is not too far from the site $x=0$ because for larger distances the kink translational mode changes significantly and nonlinear effects appear.

Chaotic behaviour of such solitons has been previously predicted [3] and also observed [12-14]. It has been proved [13] that in a more general case when the external force $F(x)$ can possess three zeros (what is equivalent to a double well potential $V(x)$ or to the Duffing model) the chaotic soliton motion is possible. We will show that the chaos occurs even for a single well potential corresponding to the force (2) and several routes to chaos can be observed. Although our model is spatially extended we investigate only the position $x_{c}(t)$ of the soliton centre, i.e. we consider only one collective mode corresponding to the soliton motion. 
The numerical method used to solve Eq. (1) is a simple finite difference scheme. To diminish a possible influence of the energy flux which is reflected at the edge of the grid and can reach the centre of system, the total length of the implemented numerical grid $L=24$ was much larger than the soliton width $d=2$. The grid density was chosen so large (600 points) that no significant changes in the system dynamics were observed by a further increase in grid density. We found that for discrete step sizes $\Delta x=0.04, \Delta t=0.032$ the results converged. The initial condition was the solution (3) while boundary conditions defined for $x= \pm 12$ were

$$
\frac{\partial \varphi}{\partial x}=0
$$

\section{Routes to chaos}

The Feigenbaum scenario of the route to chaos was observed when we increased the amplitude $P$ of the force $G(x, t)$ by fixed values of other parameters $B=0.53, \gamma=0.15, \omega_{0}=0.36$. Oscillations of the soliton centre $x_{\mathrm{c}}(t)$ exhibit a characteristic sequence of period doublings and are presented in Fig. 1. From the resulting bifurcation diagram (Fig. 2) we estimated the characteristic Feigenbaum number $\delta \approx 4.8 \pm 0.4$.
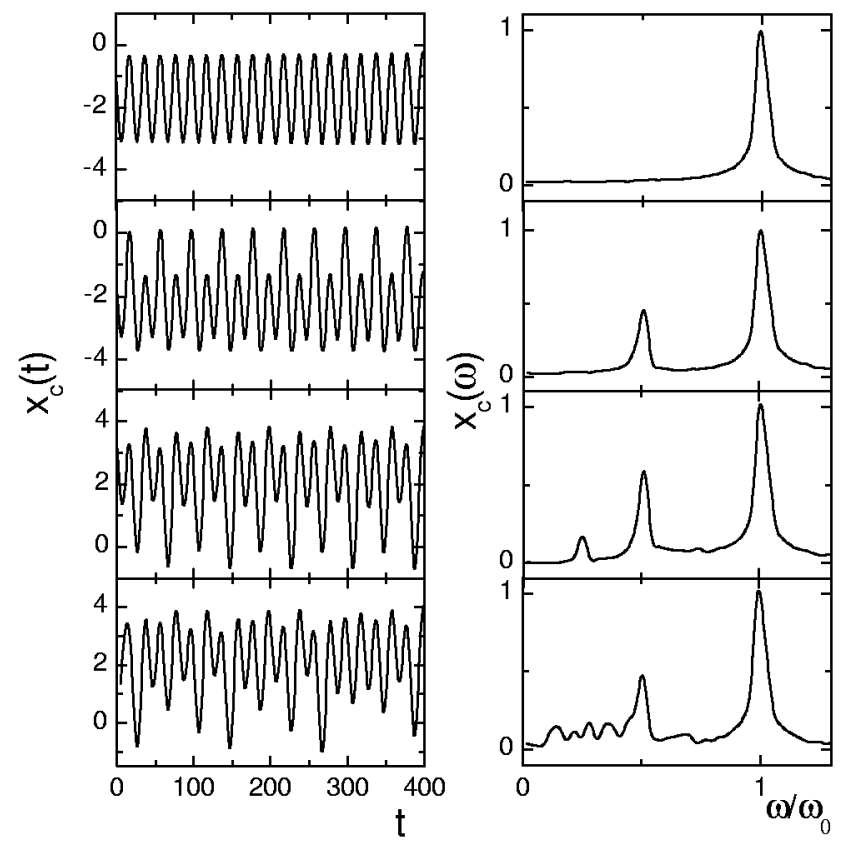

Fig. 1. Time series and power spectra for the motion of soliton centre when $B=0.53$, $\gamma=0.15, \omega_{0}=0.36$, and $P=0.26,0.32,0.343$, and 0.36 , from top to bottom, respectively. 


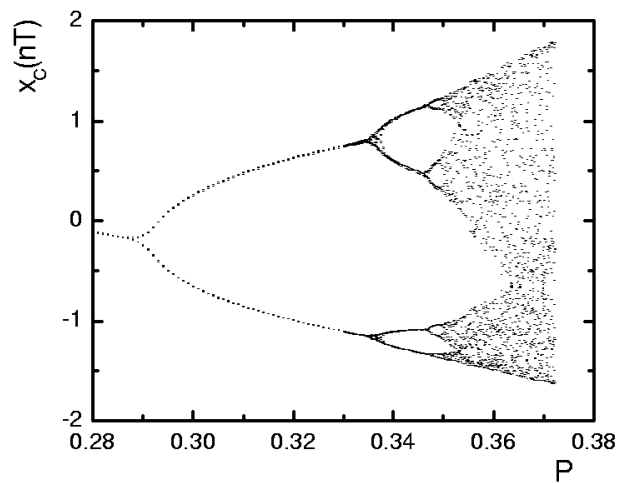

Fig. 2. Bifurcation diagram for the amplitude of soliton oscillations. Parameter values as for Fig. 1, $T$ is the period of the pumping force $G$.

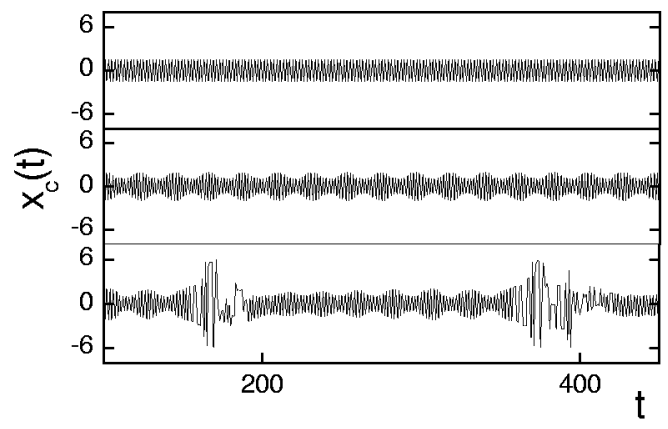

Fig. 3. Time series of the soliton centre $x_{c}(t)$ for parameter values $B=0.55, \gamma=0.05$, $\omega_{0}=0.7884$ and $P=0.265,0.296,0.3$, from top to bottom, respectively. 


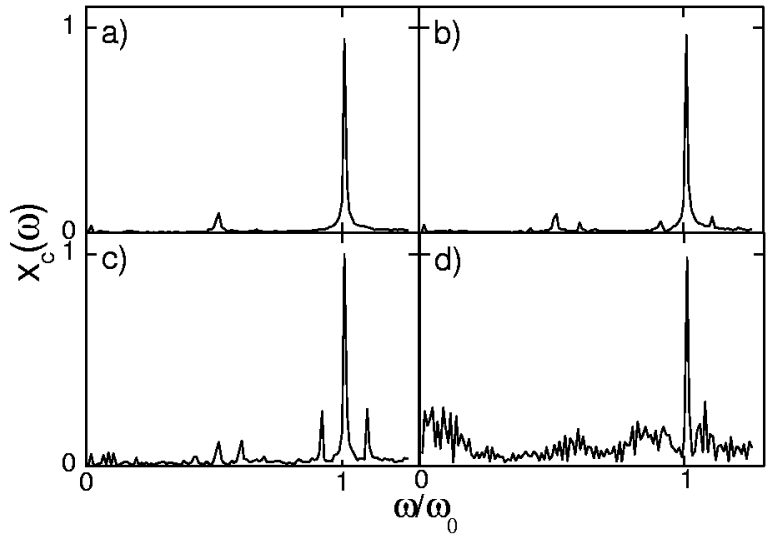

Fig. 4. Power spectra for soliton dynamics. The amplitude $P$ is: 0.265 (a), 0.28 (b), 0.296 (c), 0.3 (d), other parameters as for Fig. 3. 


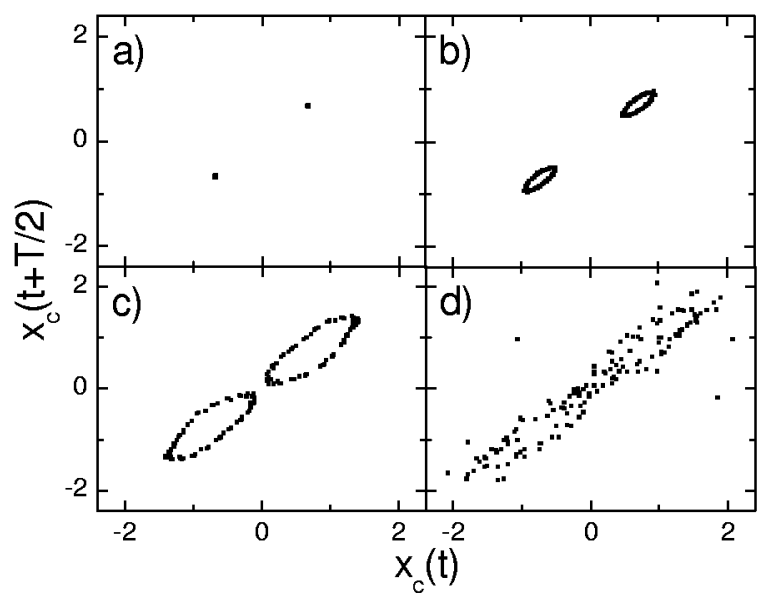

Fig. 5. Stroboscopic maps for time series for $P=0.265$ (a), 0.28 (b), 0.296 (c), 0.3 (d).

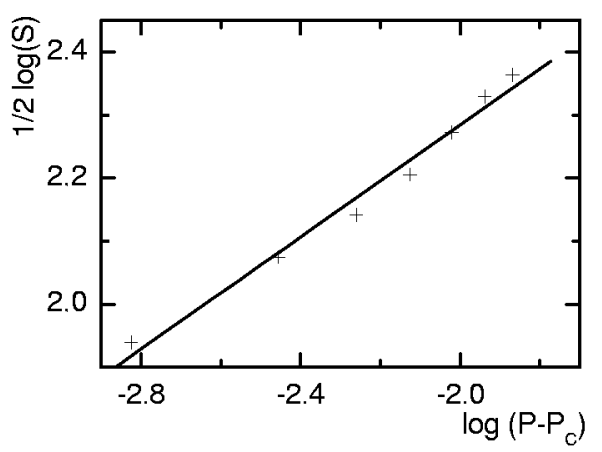

Fig. 6. The square root of the area of the ellipses of Fig. 5 vs. control parameter. The solid line is drawn for the expected exponent $\beta=1 / 2$.

Another scenario can be found when $B=0.55, \gamma=0.05, \omega_{0}=0.7884$. In such a case the increase in the parameter $P$ leads to the following path: birth of the torus via the Hopf bifurcation of the periodic solution, growth of the torus, an intermittent soliton motion and in the end the full developed chaos. Time series in Fig. 3 demonstrate the appearance of slow modulations of the amplitude of the initially nearly sinusoidal oscillations of $x_{\mathrm{c}}(t)$ above the bifurcation point $P_{\mathrm{c}}$. The critical bifurcation value was numerically estimated as $P_{\mathrm{c}}=0.2826$. At the power spectrum in Fig. 4, the new frequency $\omega_{1}$ appears as peaks for $\omega_{0}-\omega_{1}$ and $\omega_{0}+\omega_{1}$, where $\omega_{1} / \omega_{0} \sim 1 / 11$. A further increase in $P$ leads to decrease of the central hole of the torus (Fig. 5) and to decrease in the modulation frequency $\omega_{1}$. Simultaneously the changes of $P$ amplify the modulation amplitude which can be observed in Fig. 5. The square root of ellipse surface seen in Fig. 5 and calculated as a function of the control parameter $P$ is shown in Fig. 6. The result resembles 


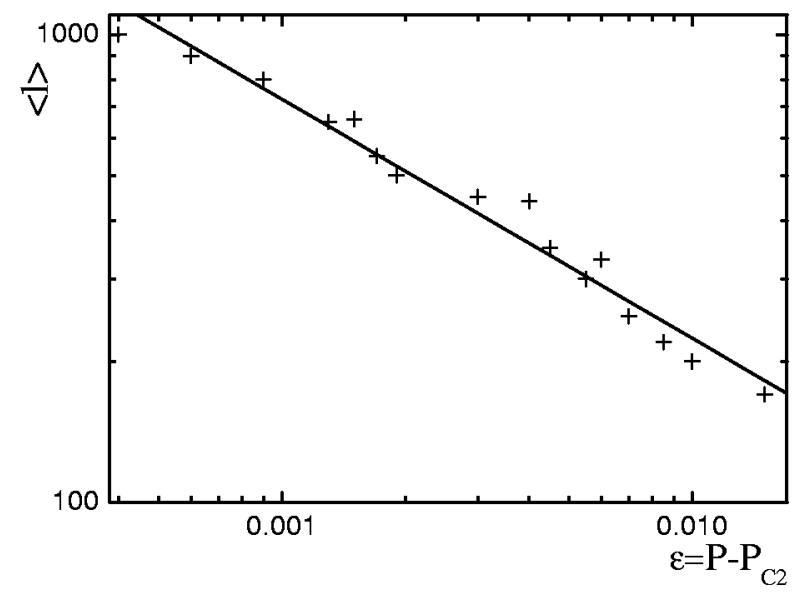

Fig. 7. The average length of laminar regimes vs. distance from the critical point.

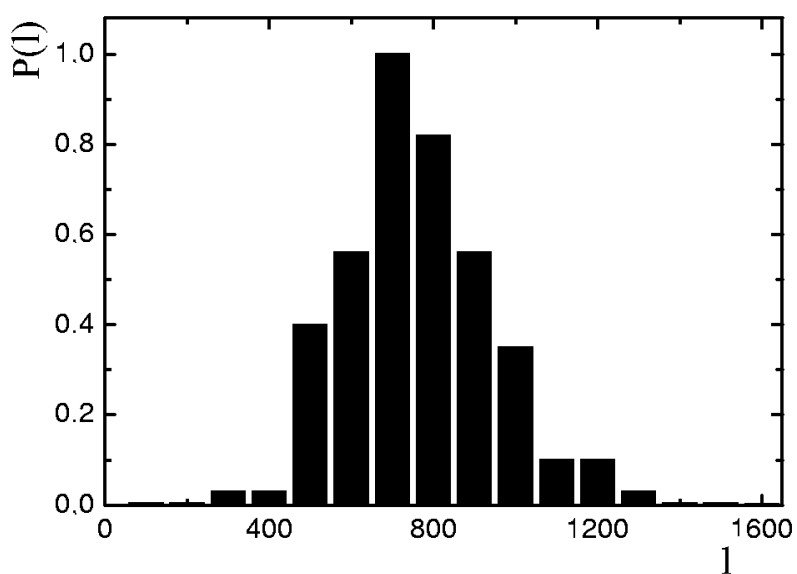

Fig. 8. The histogram of the length of regular oscillation near the intermittency threshold.

the so-called square root law that occurs for the secondary Hopf bifurcations [24]

$$
\sqrt{S} \sim\left(P-P_{\mathrm{c}}\right)^{\beta}
$$

where $\beta=1 / 2$ and in our model we obtained the exponent $\beta=0.43 \pm 0.04$. At the moment when the central hole of the torus vanishes a new phenomenon appears. The quasiperiodic motion is now aperiodically interrupted by erratic bursts of large amplitude oscillations (Fig. 3). The behaviour looks similar to the chaotic intermittency and to specify the intermittency type we investigated the scaling law for average lengths of laminar regimes (Fig. 7). The obtained exponent $\gamma=$ $-0.45 \pm 0.03$ is close to the characteristic value $\gamma=-0.5$ for the intermittency of type I [25]. Figure 8 presents the histogram of lengths of laminar phases (regular oscillations) near the intermittency threshold, which is also characteristic of this 


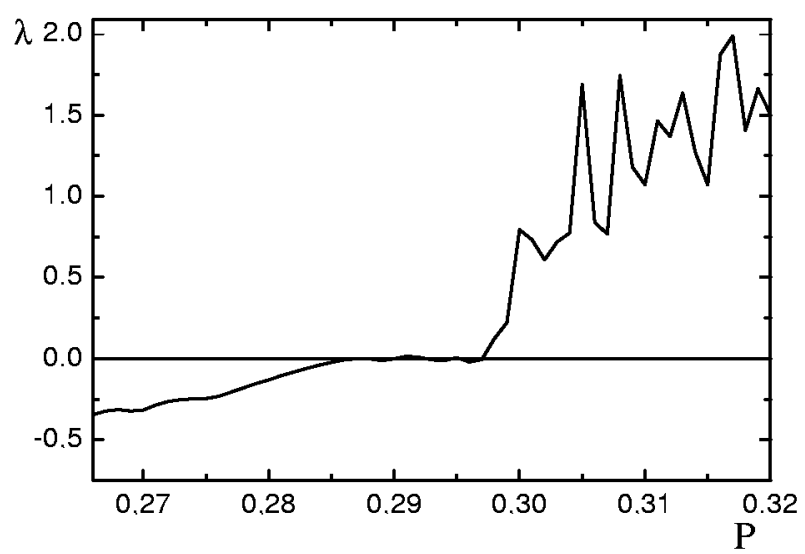

Fig. 9. The maximal Lyapunov exponent vs. control parameter. Three regimes are clearly seen: $\lambda<0, \lambda \simeq 0, \lambda>0$.

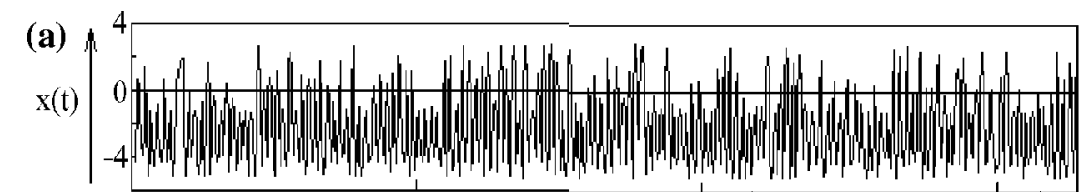

(b)
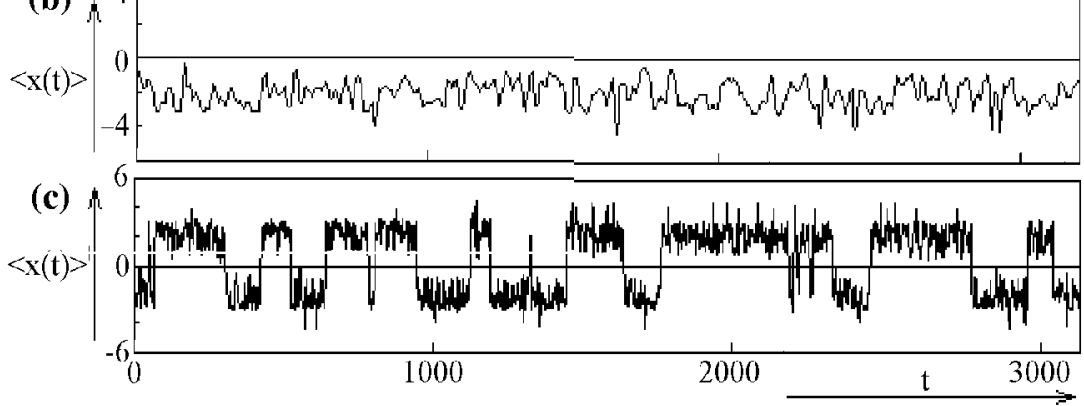

Fig. 10. Time series for soliton centre of mass (a). System parameters: $P=0.35$, $B=0.53, \gamma=0.15, \omega_{0}=0.3$. A symmetric trajectory $x(t) \rightarrow-x(t)$ was observed by other initial conditions; (b) time average of the trajectory (a); (c) averaged trajectory for $P=0.37$.

type of intermittency. Finally, this transition to chaos is illustrated in Fig. 9, where the largest Lyapunov exponent versus the control parameter is depicted. Similar behaviour of spatially dependent systems was observed for Belousov-Zabotynski chemical reaction [26].

The last observed phenomenon was the chaos-chaos intermittency [27-29]. Below the critical value of the control parameter $P$ the kink performs chaotic oscillations around a certain position which is located either to the left or to the right of the system centre $x=0$ depending on initial conditions. An example 


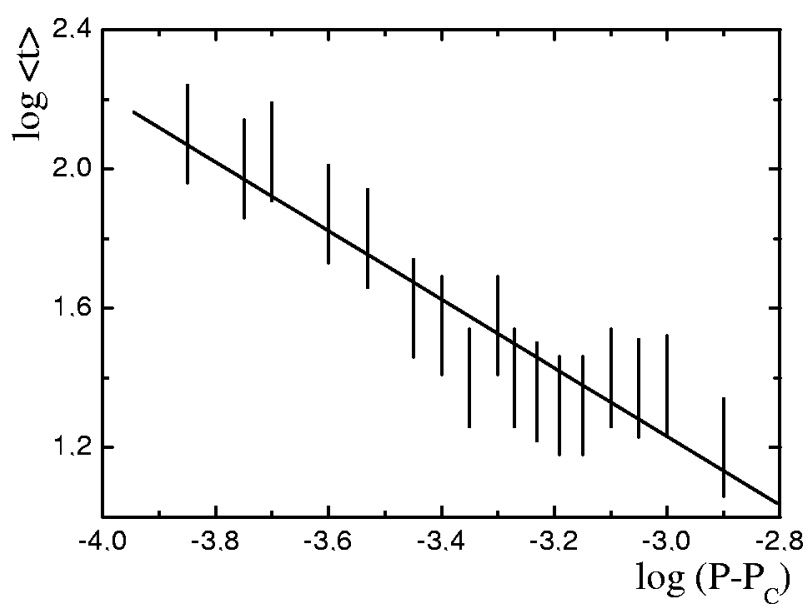

Fig. 11. Average time between jumps of the soliton from one chaotic attractor to another.

of such a motion is presented in Fig. 10a. The phenomenon is easier to observe when the time averaging is applied (Fig. 10b). It follows that in the system there are two distinct chaotic attractors corresponding to the motion of the kink on the left or on the right side of the phase boundary. If the control parameter $P$ increases the both attractors merge at the critical point. For higher values of $P$ the chaos-chaos intermittency appears [30] (Fig. 10c), i.e. the soliton jumps in a chaotic way between two different parts of the chaotic attractor. The observed critical exponent $\gamma$ describing the scaling of the average time $\tau$ between such jumps is not universal because it depends on the Lyapunov exponents of the mediated orbit $[29,31]$. In our case it equals to $\gamma=1.1 \pm 0.1$ (Fig. 11).

\section{Chaos synchronization}

To observe the synchronization of two chaotic solitons we consider a model of two coupled one-dimensional systems described by Eq. (1) with indices 1 and 2, respectively. The coupling is unidirectional and is introduced by adding the following term to the first system:

$$
f_{s}\left(x^{(1)}, t\right)=\frac{K\left[x_{c}^{(2)}(t)-x_{c}^{(1)}(t)\right]}{\cosh ^{2}\left(B x^{(1)}\right)}
$$

where $x_{c}^{(1)}$ is the centre of the first soliton and $x_{c}^{(2)}$ is the centre of the second one. Figure 12 shows the averaged distance between both centres as a function of the amplitude $K$ of the synchronizing force. Let us start with no synchronizing force ( $K=0$ ) and taking as the initial condition the configuration when both solitons are located on different sides of the phase boundary, i.e. on separated chaotic attractors. When the coupling parameter $K$ gradually increases, the synchronizing 


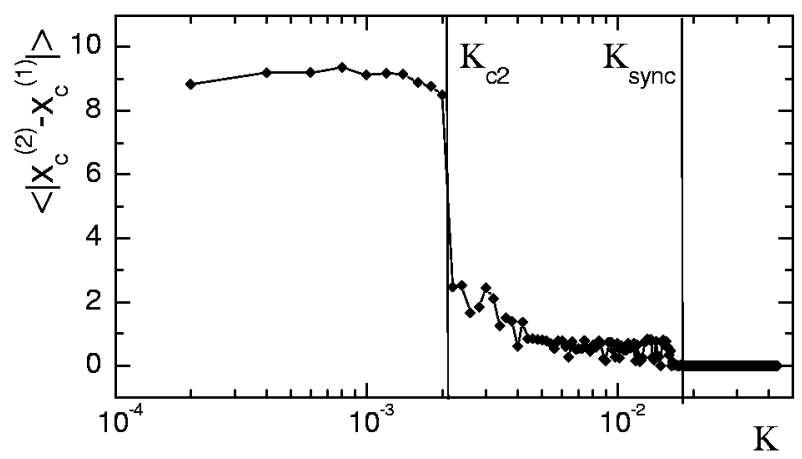

Fig. 12. Averaged distance between centres of solitons as a function of the amplitude $K$ of the synchronising force.

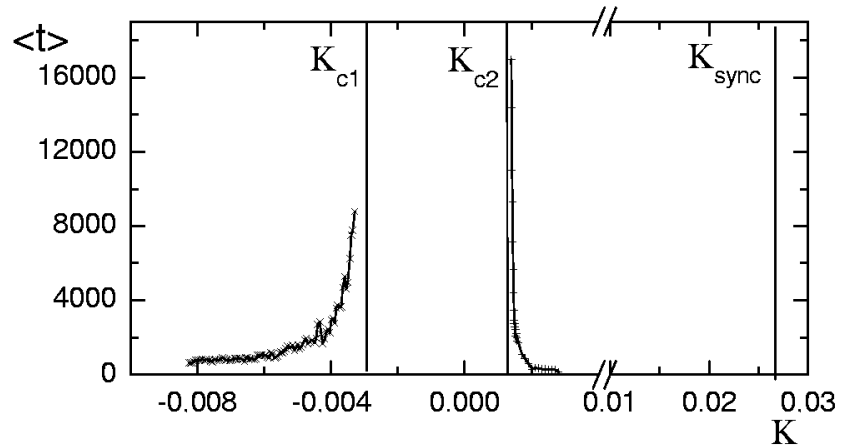

Fig. 13. The average time needed for a soliton jump from one attractor to another as a function of the parameter $K$.

force is at first too weak to shift the soliton (1) to the attractor of the soliton (2), i.e. to induce a jump of the soliton over the phase boundary. A nonzero probability of such a jump is only for $K>K_{\mathrm{c} 2}$. Figure 13 shows the inverse of this probability, i.e. the averaged transient time before such a jump takes place. This transient time decreases very fast with the increase in the parameter $K$. It is important to stress that there is a region of coupling constants $K_{\mathrm{c} 2}<K<K_{\text {sync }}$ when the soliton (1) can be shifted to the attractor of the soliton (2) and both solitons move chaotically on the same attractor but they are not synchronized. Only for much higher values of the coupling parameter $K>K_{\text {sync }}$ (where $K_{\text {sync }} \sim 10 K_{\mathrm{c} 2}$ ) the synchronization takes place. The corresponding partial (relative) Lyapunov exponent $A_{\text {rel }}$ measured from the observation of the distance between soliton centres $x_{c}^{(2)}(t)-x_{c}^{(1)}(t)$ changes a sign (because it is the transition inside chaos, the largest Lyapunov exponent of the considered system remains positive) [30]. Figure 14 shows the averaged behaviour of $\Lambda_{\text {rel }}$ as a function of the coupling constant. One can see that there is only a limited range of the parameter $K$ when the synchronization is possible and inside this region a characteristic minimum of the relative Liapunov 


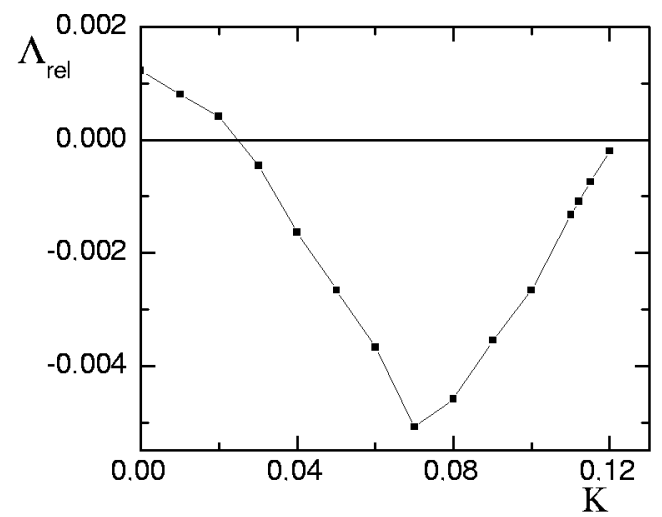

Fig. 14. Averaged range of the full synchronization.

exponent exists. It is interesting that such behaviour is similar to the phenomenon observed for chaos control by time-delayed feedback [19, 20, 32]. If the solitons are synchronized and the coupling constant $K$ is decreased then for $K=K_{\text {sync }}$ the synchronization disappears. But even for $K=0$ the both solitons move always on the same attractor. Only when $K$ is negative and smaller than another critical constant $K_{\mathrm{c} 1}$ the repelling force between solitons is large enough and there is a nonzero probability of a jump of the soliton (1) to another attractor (see Fig. 13). It follows that there is a hysteresis in the system behaviour.

\section{Conclusion}

We have investigated a highly nonlinear spatially extended system: one-dimensional $\varphi^{4}$ model [21] in the presence of a phase boundary and a pumping force. Due to the presence of spatially localized solutions in the form of kink solitons the resulting routes to chaos in this system resemble typical features of models described by ordinary differential equations or low-dimensional maps, i.e. the Feigenbaum cascade, intermittency of type I, and chaos-chaos intermittency. Synchronization of two such chaotic systems is connected with the hysteresis phenomenon.

\section{Acknowledgments}

Authors are grateful to Wolfram Just for helpful discussion. One of us (JAH) is thankful to Dirk Helbing and Holger Kantz for their hospitality during the stay in Dresden. The work has been in part supported by the State Committee for Scientific Research grants 2 P03B 03518,3 T09C 037 16, and by the ALTANA AG due to the Herbert Quandt-Programm.

\section{References}

[1] Y.S. Kivshar, B.A. Malomed, Rev. Mod. Phys. 61, 763 (1989).

[2] A.R. Bishop, D.K. Campbell, St. Pnevmatikos, Disorder and Nonlinearity, Springer-Verlag, Berlin 1989. 
[3] J.A. Gonzalez, J.A. Holyst, Phys. Rev. B 45, 10338 (1992).

[4] J.A. Gonzalez, B. de Mello, Phys. Scr. 54, 14 (1996).

[5] J.A. Holyst, Phys. Rev. E 57, 4786 (1998).

[6] G. Kalbermann, Phys. Rev. E 55, R6360 (1997).

[7] Hai Wen-Hua, Chin. Phys. 9, 175 (2000).

[8] A.G. Maksimov, V.I. Nekorkin, M.I. Rabinovich, Int. J. Bifurcation and Chaos 5, 491 (1995).

[9] J. Boyce, R.Y. Chiao, Phys. Rev. A 59, 3953 (1999).

[10] A.A. Zabolotskii, Zh. Eksp. Teor. Fiz. [Sov. Phys. JETP] 119, 434 (2001).

[11] T. Nagasawa, Physics of Plasmas 6, 3471 (1999).

[12] J.A. Gonzalez, L.E. Guerrero, A. Bellorin, Phys. Rev. E 54, 1265 (1996).

[13] L.E. Guerrero, A. Bellorin, J.R. Carbo, J.A. Gonzalez, Chaos, Solitons and Fractals 10, 1491 (1999).

[14] J.A. Gonzalez, B. de A. Mello, Phys. Lett. A 219, 226 (1996).

[15] Zhang Ze Lan, Zeng Li Bo, Commun. Theor. Phys. 32, 327 (1999).

[16] T. Sameshima, K. Fukushima, T. Yamada. Physica D 150, 104 (2001).

[17] P. Fronczak, J.A. Holyst, Phys. Rev. E 65, 26219 (2002).

[18] K. Pyragas, Phys. Lett. A 170, 421 (1992).

[19] W. Just, E. Reibold, H. Benner, K. Kacperski, P. Fronczak, J. Holyst, Phys. Lett. A 254, 158 (1999).

[20] W. Just, E. Reibold, K. Kacperski, P. Fronczak, J.A. Holyst, H. Benner, Phys. Rev. E 51, 5045 (2000).

[21] J.H. Krumhansl, J.R. Schrieffer, Phys. Rev. B 11, 3535 (1975).

[22] A.R. Bishop, J.A. Krumhansl, S.E. Trullinger, Physica D 1, 1 (1980).

[23] J.A. Holyst, H. Benner, Phys. Rev. E 52, 4583 (1995).

[24] G. Iooss, W.F. Langford, Elementary Stability and Bifurcation Theory, Springer-Verlag, Berlin 1980.

[25] E. Ott, Chaos in Dynamical Systems, Cambridge University Press, Cambridge 1993.

[26] F. Argoul, A. Arneodo, P. Richetti, J.C. Roux, J. Chem. Phys. 86, 6 (1987).

[27] C. Grebogi, E. Ott, J.A. Yorke, Physica D 24, 243 (1987).

[28] J.A. Hołyst, A. Sukiennicki, Acta. Phys. Pol. A 81, 353 (1992).

[29] K. Kacperski, J.A. Holyst, Phys. Rev. E 55, 5044 (1997).

[30] L.M. Pecora, T.L. Carrol, Phys. Rev. Lett. 64, 821 (1990).

[31] C. Grebogi, E. Ott, F. Romeiras, J.A. Yorke, Phys. Rev. A 36, 5365 (1987).

[32] W. Just, M. Ostheimer, E. Reibold, H. Benner, Phys. Rev. Lett. 78, 203 (1997). 